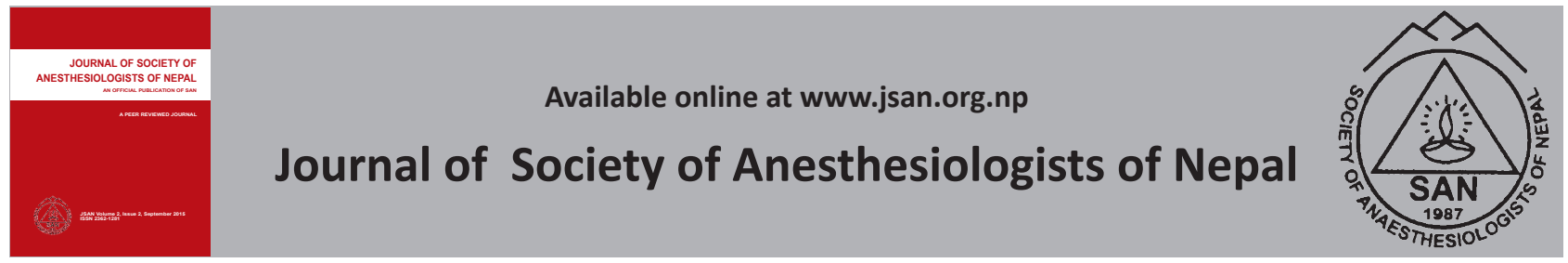

Case Report

\title{
Anesthetic and critical care challenges in massive chyle leak following robotic surgery: a special case report
}

Uma Hariharan $^{ \pm}$, Itee Choudhary ${ }^{*}$, Ajay Kumar Bhargava ${ }^{*}$

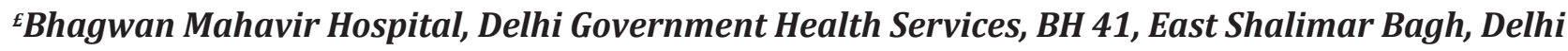

110088, India

${ }^{*}$ Rajiv Gandhi Cancer Institute and Research Centre, Sector 5, Rohini, Delhi 110085, India

\author{
ARTICLE INFO \\ Article History \\ Received 05.03.2015 \\ Accepted 16.080.2015 \\ Published 10.09.2015 \\ (C) Authors retain copyright \\ and grant the journal right \\ of first publication with the \\ work simultaneously licensed \\ under a Creative Commons \\ Attribution License that allows \\ others to share the work with \\ an acknowledgement of the \\ workss authorship and initial \\ publication in this journal.
}

\begin{abstract}
Abstract: A unique case report is hereby presented which entails the multi-modality management of a very rare complication of modern minimally-invasive robotic surgery. Chyle leak can be an extremely challenging problem following certain surgeries, especially in cancer patients. Medical, nutritional and/or surgical management is required to control the leak. Prolonged massive chyle leaks which do not respond to conservative management require surgical intervention. Retroperitoneal lymph node dissection is a complex procedure undertaken for lymph node clearance in germ cell tumors after primary radical surgery. Robotic surgeries have revolutionized the field of minimally invasive operations. A patient who underwent robotic nerve sparing retroperitoneal lymph node dissection for non-seminomatous germ cell tumor of the right testis, post-chemotherapy, developed a continuous large volume chylous ascites, presenting with electrolyte imbalances and hypotension. Exploratory laparotomy in the operation theatre was required for sealing the chyle leak after failure of medical and nutritional management in the intensive care unit. The main take-away message is that refractory chyle leak can occur in retroperitoneal surgeries requiring multidisciplinary management. Careful coordinated action between critical care, oncological, anesthesia and surgical teams is mandatory for a favourable outcome. Familiarity with the nuances of robotic surgery and eternal vigilance are the keys to successful conduction of complex robotic procedures.
\end{abstract}

Keywords: Chyle; germ cell cancer; laparotomy; lymph node dissection; remote operations robotic; retroperitoneal space

How to cite this article: Hariharan U, Choudhary I, Bhargava AK. Anesthetic and critical care challenges in massive chyle leak following robotic surgery: a special case report. JSAN 2015;2:73-76..

Corresponding author: Dr Uma Hariharan (MBBS, DNB, PGDHM, Fellowship Oncoanaesthesia),

Specialist Anesthesia, Delhi Government Health Services.

BH 41, East Shalimar Bagh, Delhi 110088, India

Ex-Fellow Rajiv Gandhi Cancer Institute and Research Centre,

Sector 5, Rohini, Delhi 110085, India

Mobile: $+91-9811271093$

Email:uma1708@gmail.com 


\section{Introduction}

Robotic surgery is the future of the minimal access surgery era. It provides a three dimensional view to the surgeon with seven degrees of freedom and dexterity similar to the human hand. It is associated with improved patient outcome in terms of less blood loss, quicker recovery and less postoperative pain. But it has a steep learning curve. Anesthetic concerns include, steep trendelenberg position with its attendant complications, prolonged pneumoperitoneum, judicious fluid therapy and difficulty in quick de-docking during emergency situations. ${ }^{1}$ From surgical point of view, robotic surgery may cause blood loss which may be difficult to estimate (torrential haemorrhage needs to be guarded against, if working close to major vessels), nerve and lymphatic damage. Chylous ascites is the leakage of the lipid-rich lymph into the peritoneal cavity. ${ }^{2}$ Chyle leak can occur as a complication due to damage to major lymphatic vessels, which can occur following both open and robotic surgeries. Cisterna chyli is a lymphatic sac in the abdomen situated between the great vessels, which can get damaged in any retroperitoneal surgery, especially in lymph node dissections. ${ }^{3}$ If not corrected, chyle leaks can lead to multiple problems (metabolic, immunological, physical and nutritional), leading to increased patient morbidity and mortality.

We present an outline of the anesthetic course of events and intensive care management of massive chyle leak following robotic retroperitoneal lymph node dissection in a cancer patient.

\section{Case Presentation}

A 20 year old, ASA grade I male patient was posted for right high inguinal orchidectomy for Non-Seminomatous Germ Cell Tumor of the right testes (Stage 3c NSGCT). This was followed by 4 cycles of chemotherapy with Bleomycin, Etoposide and Cisplatin (BEC). Post-chemotherapy, CT (computed tomography) scan of the abdomen showed residual disease in the right retroperitoneal lymph nodes (enlarged aorto-caval lymph nodes). CECT (contrastenhanced (T) of the chest showed metastatic deposits in the right lung. CT scan of the brain was unremarkable. Patient was again posted for robotic retroperitoneal lymph node dissection (nerve sparing) and right lung metastatectomy (open postero-lateral thoracotomy) under general anesthesia. All standard monitors were instituted, along with invasive monitoring (arterial and central venous pressure catheters), as well as neuromuscular monitor, multi-gas monitor, airway pressure monitor and BIS (bi-spectral index). After intubation with a single lumen tube (SLT), steep trendelenberg positioning and carbondioxide pneumo-peritoneum was done for docking of the robot. During robotic surgery, there was evidence of lymph node enlargement in inter-aortocaval and paracaval regions, which were dissected out. After de-docking, pneumoperitoneum was released and patient was turned supine from extreme head down position. SLT was replaced with a 39 French left-sided Mallinckrodt ${ }^{\mathrm{TM}}$ [Mallinckrodt Inc, St Louis, MO, USA] Double lumen tube (DLT) for one lung ventilation (OLV) and its position confirmed by fibreoptic bronchoscopy (FOB). During right postero-lateral thoracotomy, 9 metastatic nodules were removed (3 from right lower lobe and 6 from right upper lobe). After thoracic surgery, OLV was discontinued, patient turned to supine position and extubated using standard guidelines. Patient recovered well in the onco-surgical intensive care unit. Both the above surgeries were uneventful and the patient was discharged later with normal blood and other investigations.

One month later, the patient presented to the emergency department with fever, hypotension, vomiting and abdominal distension. He gave history of weight loss (20 Kilograms in one month) and decreasing urine output. On examination, the patient was emaciated, with tense abdomen and fluid thrill. He had electrolyte imbalance in the form of hyponatremia (Serum Na+ $114 \mathrm{mEq} / \mathrm{L}$ ) and hypocalcemia (serum calcium $8 \mathrm{mg} / \mathrm{dL}$ ). He also had mild leucocytosis (TLC 10,100/mm3), raised serum creatinine $(1.67 \mathrm{mg} / \mathrm{dL})$ and severe hypoalbuminemia (serum albumin level $1.5 \mathrm{gm} / \mathrm{dL}$ ). Chest X-ray revealed mild right-sided pleural effusion, with normal lung fields. $X$-ray of the abdomen revealed gaseous distension without air-fluid levels. USG abdomen suggested ascites with normal liver, pancreas, spleen, kidneys and bladder. Patient was able to pass flatus and on auscultation, bowel sounds were present. After initial resuscitation and supportive treatment, urine output and creatinine levels improved. Medical management was done for electrolyte disturbances, in the form of extra-salt diet, normal saline I.V infusion, oral selective vasopressin receptor-2 antagonist (15mg Tolvaptan OD) for hyponatremia as well as slow I..V Calcium Gluconate (10\%) infusion for hypocalcemia. Urine output improved with fluid resuscitation and I.V. furosemide therapy. CT guided abdominal pig-tail catheter insertion was done under local anesthesia and drain connected to a graduated urobag. Immediately, $1000 \mathrm{ml}$ of turbid, light yellow ascitic fluid drained over a period of one hour. The amount of drain increased with time and a total of $9000 \mathrm{ml}$ of chylous fluid was drained over 24 hours. This fluid was sent for biochemical analysis, which proved it to be chyle (Milky fluid with triglyceride levels $325 \mathrm{mg} / \mathrm{dL}$ and leukocyte count of 1050 cells $/ \mathrm{mm}^{3}$ ). It was decided to give a trial of conservative management for chylous ascites. Nutritional support in the form of albumin and essential fatty acid I.V infusions along with oral medium chain fatty acids with high protein diet were started. Even after medical correction of electrolyte imbalances, intravenous broad spectrum antibiotics and nutritional therapy, chylous acites remained unabated. Daily chyle output was around 8-9 litres. After 1 week of conservative therapy, it was decided to surgically correct the chyle leak. In order to easily detect the site of leak during laprotomy, 200grams of melted butter was given through the ryle's tube 2 hours prior to surgery. Preinduction, lower thoracic epidural 
catheter placement was done under local anesthesia for peri-operative analgesia. This was followed by standard monitoring, aspiration prophylaxis, general anesthesia with rapid sequence induction, cricoid pressure, endotracheal intubation and controlled mechanical ventilation. Dose adjustments were made in view of weight loss and hypoalbuminemia (for protein- bound drugs). On opening the abdomen and retroperitoneum, chyle leak was noted from aberrant lymphatic duct, just lateral to the aorta and below the left renal vein. Another small lymphatic leak was noted in the inter-aortocaval region, above the left renal vein. Both the aberrant lymphatic ducts were sutured with 5-0 prolene sutures and hemostasis obtained before abdominal closure. Trachea was extubated at the end of the procedure and shifted to the onco-surgical intensive care unit. Postoperative pain management was accomplished with epidural bupivacaine and fentanyl infusion. Patient maintained all vital parameters in the entire peri-operative period. Chyle leak through the drain became negligible and completely dried by the $2^{\text {nd }}$ postoperative day. Patient was shifted out of ICU after 48 hours, when all his investigations normalized and was managed in ward with nutritional and nursing support. Postoperative serum levels of electrolytes were also within normal limits. He also started gaining weight after 1 week, when he was discharged home with advice for regular follow-up with the onco-surgeons and nutritionist.

\section{Discussion}

Retroperitoneal lymph node dissection can lead to damage to nerve ${ }^{4}$, vessel or lymphatics. Germ cell tumors of the testes can have hematogenous and lymphatic dissemination. ${ }^{5}$ In our case, after the primary surgery, patient underwent dissection of involved lymph nodes in retroperitoneum robotically, followed by thoracotomy for excision of metastatic lung nodules. Both these surgeries were uneventful. Cisterna chyli would have been damaged during retroperitoneal surgery ${ }^{3}$, leading to slow lymphatic leakage, resulting in chylous ascites. ${ }^{2}$ This continuous leakage of chyle caused weight loss and other metabolic abnormalities. Correction of electrolyte abnormalities was also a challenge. Selective vasopressin receptor antagonists are useful in cases of refractory hyponatremia. ${ }^{6}$ They cause increase in urine water excretion that results in an increase in free water clearance (aquaresis), a decrease in urine osmolality, and a resulting increase in serum sodium concentration. In our case, the patient responded to oral Tolvaptan and hyponatremia was slowly corrected. Patient was given a trial of conservative management for his massive chyle leak, along with supportive nutritional therapy. ${ }^{7}$ Since this failed to control the leak, surgical correction was contemplated. The volume of chyle leak was also high enough to warrant a surgical intervention.

Preoperatively, fat in the form of melted butter was given through the ryles tube, in order to help the surgeons identify the site of chyle leak intra-operatively. Hence, rapid sequence induction with cricoid pressure was done to prevent aspiration. Once the site of aberrant lymphatic drainage was identified and sutured, chyle leakage stopped. The rest of the perioperative course was uneventful. Even though robotic surgery has a lot of advantages, one needs to be careful while dissecting along neurovascular planes. Robot-assisted nerve sparing dissection was done in our case to preserve his erectile (prevent retrograde ejaculation) function. ${ }^{8}$ Greater care needs to be exercised while operating in the retroperitoneal area, as it not only houses the lymphatics but also the aorta and the inferior vena cava. Chyle leaks sometimes stop with conservative and nutritional therapy. They could also respond to somastostatin therapy. ${ }^{9}$ Somastostatin was not used in our case as it can cause hypotension and our patient presented with hypotension and electrolyte imbalance. However, a somatostatin analogue, Octreotide is the agent most commonly employed, beginning with a dose of 50 mcg thrice a day (TID) given subcutaneously, upto a maximum of $200 \mathrm{mcg}$ TID. Different infusion regimens are also available. It suppresses the secretion of several hormones, including growth hormone, glucagon, insulin and gastrointestinal hormones (gastrin, motilin, secretin and pancreatic polypeptide). The purported mechanism of action in chyle leak is mainly by deceleration in lymph flow which facilitates closure of the leak as well as by decreasing splanchnic blood flow. Its main side effects include abdominal pain, nausea, flatulence, hyper-or hypoglycemia, headache, orthostatic hypotension, visual and, bowel disturbances.

\section{Conclusions}

Retroperitoneal lymph node dissection through any route can lead to damage to several important structures. Robotically-assisted approach to the retroperitoneum must be performed with proper precautions, especially while handling lympho-vascular structures. Chyle leak, though a very rare complication following robotic surgeries can be quite challenging and may not respond to conservative management. It requires a team effort and coordination between the anesthesiologist, intensivist, oncologist, surgeon, nutritionist and physiotherapist for a successful patient outcome.

\section{Conflict of Interest: nil}

\section{Financial support/sponsorship: nil}

\section{References}

1. Lee JR. Anesthetic considerations for robotic surgery. Korean J Anesthesiol 2014;66:3-11.

2. Al-Busafi SA, Ghali P, Deschenes M, Wong P. Chylous Ascites: Evaluation and Management. ISRN Hepatology, vol. 2014, Article ID 240473, 10 pages, 2014.

3. Loukas M, Wartmann CT, Louis RG, Tubbs RS, Salter EG, Gupta AA, et al. Cisterna chyli: a detailed anatomic investigation. Clin Anat 2007;20:683-8.. 
4. Tanaka T, Kitamura H, Kunishima Y, Takahashi S, Takahashi A, Masumori $\mathrm{N}$, et al. Modified and bilateral retroperitoneal lymph node dissection for testicular cancer: peri-and postoperative complications and therapeutic outcome. Jpn J Clin Oncol 2006;36:381-6.

5. The International Germ Cell Cancer Collaborative Group. International Germ Cell Consensus Classification: a prognostic factor-based staging system for metastatic germ cell cancers. J Clin Oncol 1997;15:594-603.

6. Friedman B, Cirulli J. Hyponatremia in critical care patients: frequency, outcome, characteristics, and treatment with the vasopressin V2-receptor antagonist tolvaptan. J Crit Care 2013;28:219.e1-12.

7. Parrish CR, McCray S. Nutritional Management of Chyle Leaks: An Update. Practical Gastroenterology 2011;94:12-32.

8. Coogan $\mathrm{CL}$, Hejase MJ, Wahle GR, Foster RS, Rowling RG, et al. Nerve sparing post-chemotherapy retroperitoneal lymph node dissection for advanced testicular cancer. J Urol 1996;156:1656-8

9. Collard JM, Laterre PF, Boemer F, Reynaert M, Ponlot R. Conservative treatment of postsurgical lymphatic leaks with Somatostatin-14. Chest 2000;117:902-5. 